EDU-DORA2 (Education thérapeutique et préventive face au Diabète et à l'Obésité à Risque chez l'Adulte et l'Adolescent) is being conducted in Belgium, Luxemburg and Lorraine to investigate potential working tracks for a multifactorial and multidisciplinary approach in the management of childhood obesity (Scheen et al. 2010).

doi:10.1017/S1368980012001632

\title{
Drug therapy: actuality and perspectives
}

\author{
Daniel Brasseur
}

Head of the Medical Assessors, at the General Directorate Medicine of the Federal Ministry of Public Health of Belgium

The main role of regulators is to authorize the access to safe and efficacious medicinal products. The risk-benefit of a candidate drug should be positive for a specific disease and a target population. But is overweight or obesity as such a disease? Who should potentially be treated and be prescribed pharmacological agents? When so, what is considered to be a clinically relevant drug effect? Some answers are given for adults in 'Guideline on Clinical Evaluation of Medicinal Products used in Weight Control' by the Committee for Human Medicinal Products of the European Medicines Agency and for children in the 'Paediatric Addendum Guideline on Clinical Evaluation of Medicinal Products used in Weight Control'.

The simple conclusion of this guidance could well read: 'There is some room for drug therapy in childhood obesity, namely in severe cases and for limited duration, provided that the medicinal product is harmless and its effect sustained'. If indirect evidence demonstrates the risks linked to overweight, the impact of a drastic weight control on obesityrelated complications needs to be prospectively demonstrated in clinical trial conditions to define who will really benefit from a pharmacological treatment. Ideally the clinical relevance of a treatment allowing to lose weight (or to maintain an 'ideal' weight) should translate in less complications later in life, an increased life expectancy and a better quality of life also based on improved self-esteem. In practice such a composite endpoint is not achievable in pharmaceutical trials in an acceptable time frame. More realistically, the surrogate marker is weight (loss) accepted as a primary endpoint in most clinical trials (CT). The way to conduct CT is currently driven by the experience gained from past investigations in the field. Weight losses are often transient since partial failure or full relapses are frequent. Patients should be obese as defined, and have a documented history of failing to lose weight by means of lifestyle modifications, before enrolment into the pharmacological phase of a study. Further confounding factors related or not to the drug studied may affect the outcome of trials. Therefore regulators are de facto suspicious and require a robust methodology to circumvent bias as much as possible. The lessons learnt from the past have also contributed to carefully monitor the safety of weight losing agents. The guidance recommends collecting information during the whole trial period. Particular attention should be given to adverse events signalled from adult CT. This data set should notably encompass the adverse events related to lipid profile, liver function, cardiovascular system function and rebound phenomenon.

For centrally acting anorectic agents it is recommended that special attention and monitoring is afforded to neuropsychiatric events such as depression, sleep pattern and nightmares, assessment of self-esteem, aggression or suicidality. In growing children height velocity should also be monitored and pubertal development assessed by determining Tanner stage at baseline and endpoint. Today the tools for a quicker development of pharmacological agents are available (EC Paediatric Regulation 1901/2006) and can be used for future candidate drugs.

\section{'Over' or 'bad'-nutrition?}

\section{Marie Josee Mozin}

Founder member and Honorary President of the European Club of Paediatric Dieticians

Food intake and behaviour are considered the most important etiological factor of obesity in childhood and adolescence. The efficacy of dietary treatment depends on the regard for different stages designed to help the child and his family to modify on the long term their dietary habits, behaviour and lifestyle. 